International Journal of Engineering \& Technology, $7(2.26)(2018) 53-56$
International Journal of Engineering \& Technology
SPC
Website: $w w w . s c i e n c e p u b c o . c o m / i n d e x . p h p / I J E T$
Research paper

\title{
Farm field monitoring and irrigation automation using IOT
}

\author{
M. $\operatorname{Vinoth}^{1} *$, G. Vithiya ${ }^{2}$ \\ ${ }^{1}$ Assistant Professor, M.Kumarasamy college of Engineering,India. \\ ${ }^{2}$ M.E Student, M.Kumarasamy college of Engineering, India. \\ *Corresponding author E-mail: vinothm.cse@mkce.ac.in
}

\begin{abstract}
The Agricultural farming crops are produced in low qualitydue to lack of knowledge of farmers and crops destroyed by insects. To overcome that, many implemented factors are used. The factors can be temperature,soil,humidity,etc.,In biotechnology,some techniques are used withthe help of IoT. It is mainly used to increase the crops productivity in good quality.Existing related research works on sensor cloud have primarily focused on the challenges that wireless sensor network (WSN)-based applications. A direct estimate of water use by subsurface measurements of soil water content has been limited by the high cost of reliable soil moisture sensors. Using some sensors and techniques water can be irrigated automatically.IOT plays a vital role in smart farming.By using set of specified sensors, the irrigation management can be done properly. The sensed data from the sensors and the irrigation controller are interfaced with Wi-Fi connection. In naive Bayes, by using decision making process the farmer can decide when and where to irrigate with the help of mobile application. An algorithm was developed with threshold values of temperature and soil moisture that was programmed into a microcontroller-based gateway to control water quantity.
\end{abstract}

Keywords: IOT; Nä̈ve; Sensors; WSN.

\section{Introduction}

Biotechnology is the application of scientific techniques to modify and improve plants, animals, and Micro-organisms. Agriculture biotechnology is the area of biotechnology involving application to agriculture. GMO in which genetics is altered.Biotechnology has used in many fields,like industrial areas, including health care (medical), crop production and agriculture, non-food (industrial) uses of crops and other products (e.g. biodegradable plastics, vegetable oil, biofuels), and environmental uses. In Bioinformatics is an interdisciplinary field which addresses biological problems using computational techniques.Green Biotechnology is defined as the application of biological techniques to plants with the aim of improving the nutritional quality,quality and production economics. To protect the crops from insects, various organic fertilizers are suggested by decision engine.Two sensors are used like DHT11 (temperature and humidity) and soil moisture. Raspberry pi kit is used, along with that kit the above mentioned sensors are attached. The DHT11 gives the value of humidity and temperature and the amount of water content in the soil can be get it from the soil moisture sensor.Such sensors are inserted in the field. From that sensors, the current sensed data can be collected. Then the collected sensed data is then compared with the historical data from the knowledge base. Based on the data,the user can make the decision, how much of water can be supplied to the crops. Due to the climatic condition,the water can be supplied in both automatically or periodically by the spacial temporal techniques. Hence this system can be more efficient .by using this techniques in biotechnology,the production of crop is increased.

\section{Literature survey}

H. Navarro-Hellin and J. M., et. al. (2016), here SIDSS is implemented which means Smart Irrigation Decision Support System for the supply of water.According to the decision made by the human,the irrigation process can be done accordingly. The implemented proposed system is PLSR and ANFIS of machine learning. Here the water can be irrigated in a weekly period. By using that decision support system the decision can be taken by the user according to the weather condition. In this paper, irrigation can be done for the citrus tree at the location of south east of Spain. [1] G. Nisha and J.Megala (2014) have investigated about Wireless sensor Network based where the irrigation can be done in a correct manner. Two type of sensors are used. Temperature sensor and soil sensors are kept in the farm field, from that sensors the data can be fetched. ZigBee protocol is used to control the sensed data from the sensors. To control the overall process the microcontroller are used.The camera also kept in the field to capture the affected plant image, this can be done under image processing. [2]

D.K.Sreekantha, Kavya.A, et. al. (2017)have studied that the Internet of things (IOT), where it is used in agriculture. The farmers using the precision for the growth of the crop. In IoT it collects all the climatic condition like temperature, soil moisture content,andhumidity etc., wireless sensor network it controls everything.by using the mobile phone the user can get updated details of the land field. While using that IoT it is a cost efficient. [3]

Monali Paul, K.S., et. al. (2015) have explained the crop prediction. The sowing of crops or the supply of water can be irrigated by the farmer's prediction. That prediction can't be true always, if the temperature changed, the farmer's prediction can't be applicable for the irrigation process. For that they extract the soil dataset using data mining.On the basis of predication and the soil temperature, this is 
useful for farmer's prediction. The required amount of water can be irrigated to the crops.In that classification they used K-Nearest Neighbor method. [4]

A.T.M Shakil Ahamed, Navid T., et. al. (2015)in this paper the explained the production of crops from many problem. The problem may be due to weather changes, and also in environmental issues. By using some methodology, the problem arising can be reduced to make a better crop production. And also it can be useful for comparing the original data and the historical data, accordingly the water can be supplied. This project is mainly implemented for cultivating the cereals crops in in good quality in the major areas of Bangladesh. [5]

\section{Problem statement}

- By using Arduino kit, the power consumption is not easy and most of the algorithm cannot run in this kit.

- If the values is wrong,the exact amount of water can't be

- Supplied. Therefore, the crops leads to damage.

- It does not mention, whether the water can be supplied

- Periodically or automatically.

- For improper net connection, information can't be delivered to the system properly. So the water can't be supplied regularly.

\section{Proposed system}

The idea of this paper is to implement an automatic irrigation system by sensing the water content in the soil and also the temperature valve in the farm field. The soil moisture sensor is inserted in the soil. Depending on the quality of the sensor, it must be inserted near the roots of the plant. The soil moisture sensor measures the conductivity of the soil.

When the moisture in the soil is above the threshold, then the user is need not to supply the water. When the output from the soil moisture sensor is high i.e. the moisture of the soil is less. The circuit can be used to measure the loss of moisture in the soil over time due to evaporation and intake.In this proposed system it mainly concentrate to minimize water waste and improve plant growth. The circuit is designed to work automatically and hence there is no need for any human intervention. The project is intended for small gardens and residential environment. By using an advance soil moisture sensor, the same circuit can be expanded to large agriculture fields.

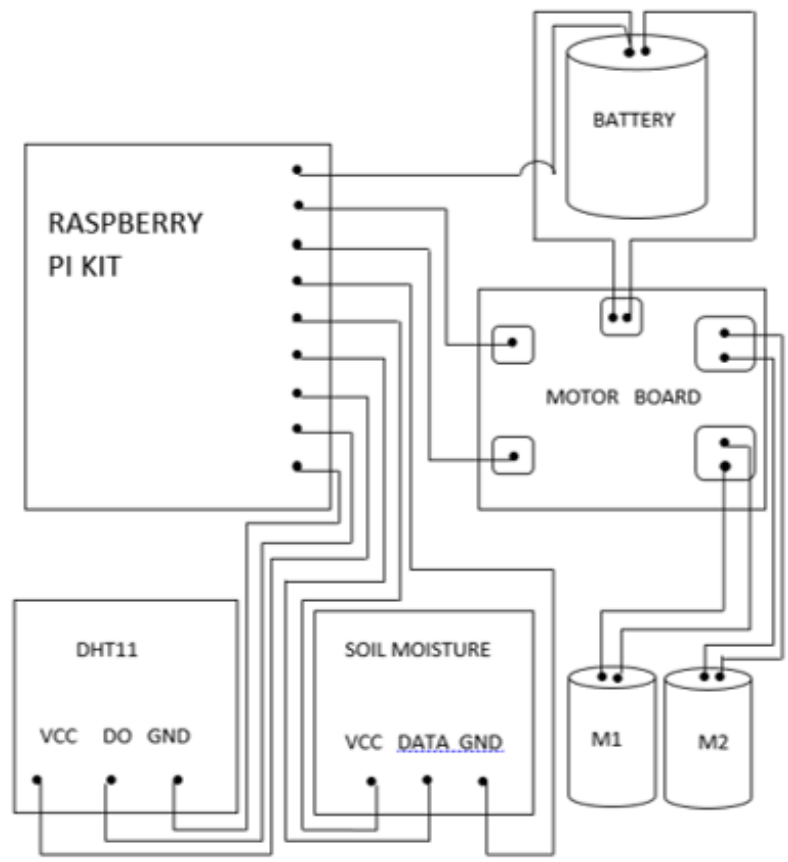

Fig. 4.1: Circuit Diagram for Crop Monitoring.

\section{Methodologies}

The learning process is divided into two types named supervised learning and unsupervised learning process. In this paradigm, when the type of output is known to the system, then it comes under the category of supervised learning. In case of unknown type output then it will be unsupervised learning model. In this machine learning paradigm, our proposed system computes the required resources in terms of water content based on the current environmental situations. This type of handling validates the current information and previous history of information which provides the exact amount of resource requirement in terms of water. In order to handle these dynamic situations, Bayesian network model is used to compute the requirement based on the current and previous history of information.

The Bayesian learning algorithm combines the features of artificial neural network and back propagation network. The ANN is used to compute the output based on the current input as well as previous history of data processed.With this concept, complete and incomplete instance separation can be done. The main advantage this approach is able to classify both complete and incomplete instances. The back propagation network model is used to update the input vectors with the precision based weighted output sequences. The sequences is a type of uncertain reasoning when the full probability model. Bayesian networks provide a natural representation for conditional independence. Bayesians interpret the a posteriori probability (output) as a measure of belief about a hypothesis or proposition updated in response to evidence. The a priori probability (input) reflects the belief about in the absence of evidence. It computes the joint distribution with the conditional independence and numerical probability.

Mean $=\underline{s 1+s 2+s 3+s 4+s 5}$

Standard deviation $=\frac{1}{n} \sqrt{\sum_{i=1}^{n}\left(X_{i}-\text { mean }\right)^{2}}$

Variance $=(\text { S.D })^{2}$

Expected value $=($ mean + variance $)$

Here the mobile application is used. While entering into the app,it contain two main division, which are CHECK STATUS and IRRIGATION. Check Status is used to check the status of the farm field.According to that value, the irrigation motor start supplying water to the field.In Irrigation, it performs Start Irrigation and Stop Irrigation. The irrigation motors turns $\mathrm{ON}$ while the user click the Start Irrigation and Stop Irrigation is used to stop the supply of water. In field of agriculture, the use of proper method of irrigation is important. The advantage of using this method is to reduce human intervention and still ensure proper irrigation.

\section{Experimental setup}

\subsection{Kit}

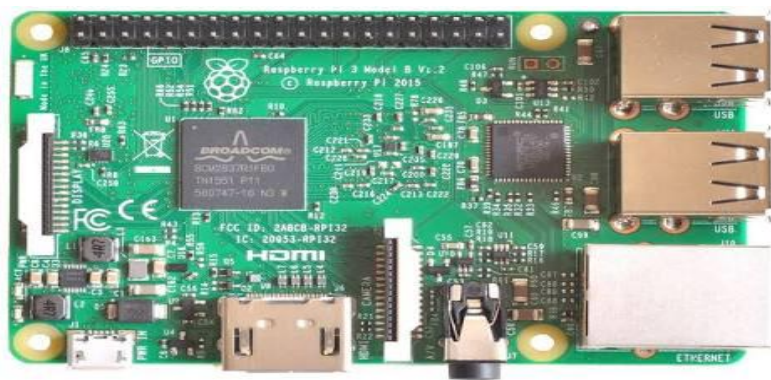

Fig. 1:.Raspberry Pi 3 


\subsection{Sensors}

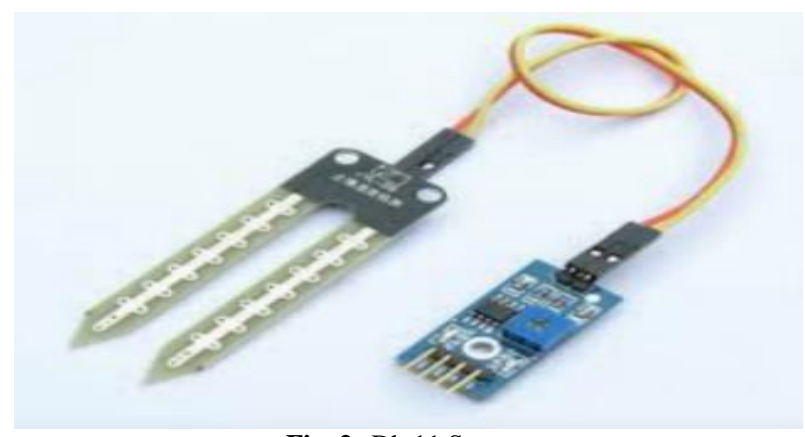

Fig. 2: Dht11 Sensor.

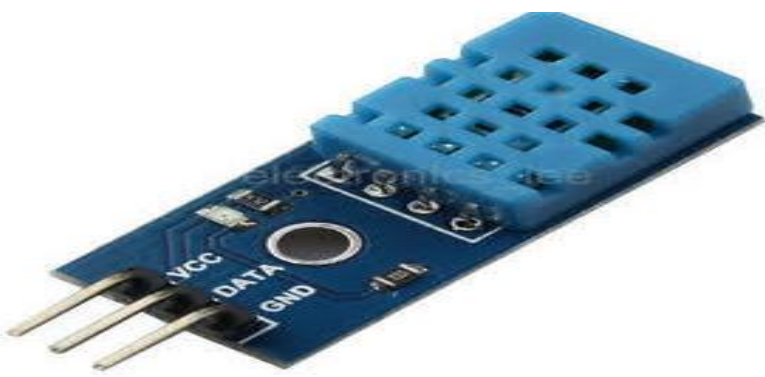

Fig. 3: Soil Moisture.

\subsection{Application}
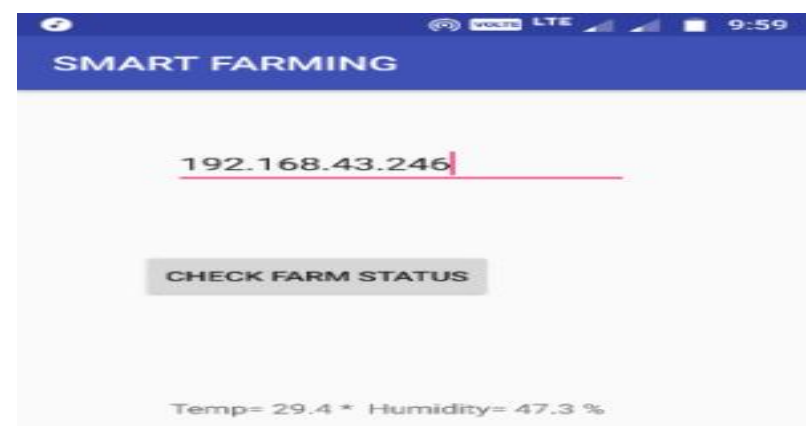

Fig. 4:.Farm Status.

\subsection{Working}

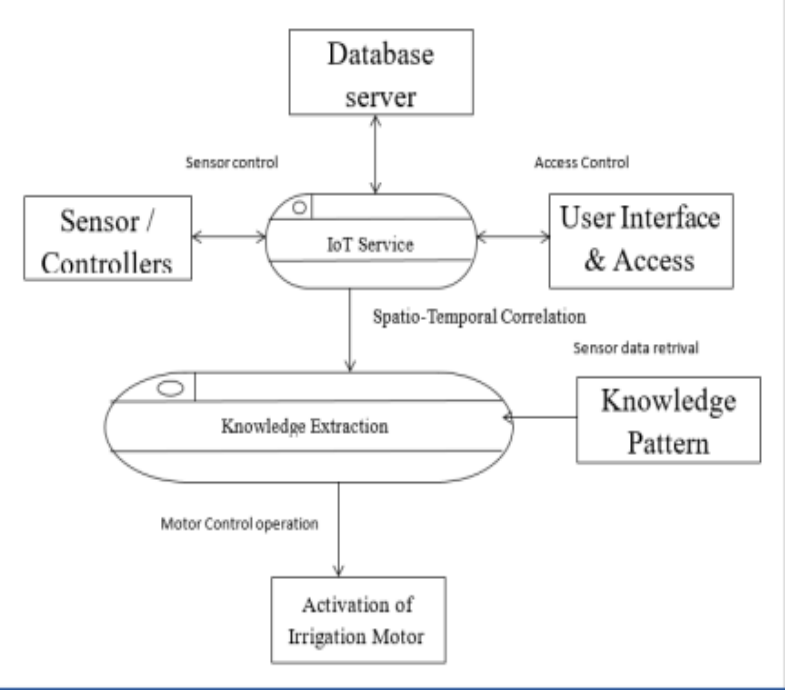

\section{Result\& discussion}

The sensor circuit, sense the data from the sensor. If the sensed data is below $5 \mathrm{v}$, then the field known to be dry. The microcontroller get the signal 1 from the timer machine, to get start the irrigation motor. If it is less than the reference voltage (ie. 5v) then the field is known to be dry, then the timer sends the logic signal 1 to the micro controller to turn ON the irrigation motor. If it is greater than the reference voltage then the timer sends the logic signal 0 to the micro controller to turn OFF the irrigation motor. Using this technique, we can reduce the communication latency and increase the rate of the access control operation.

\section{Performance analysis}
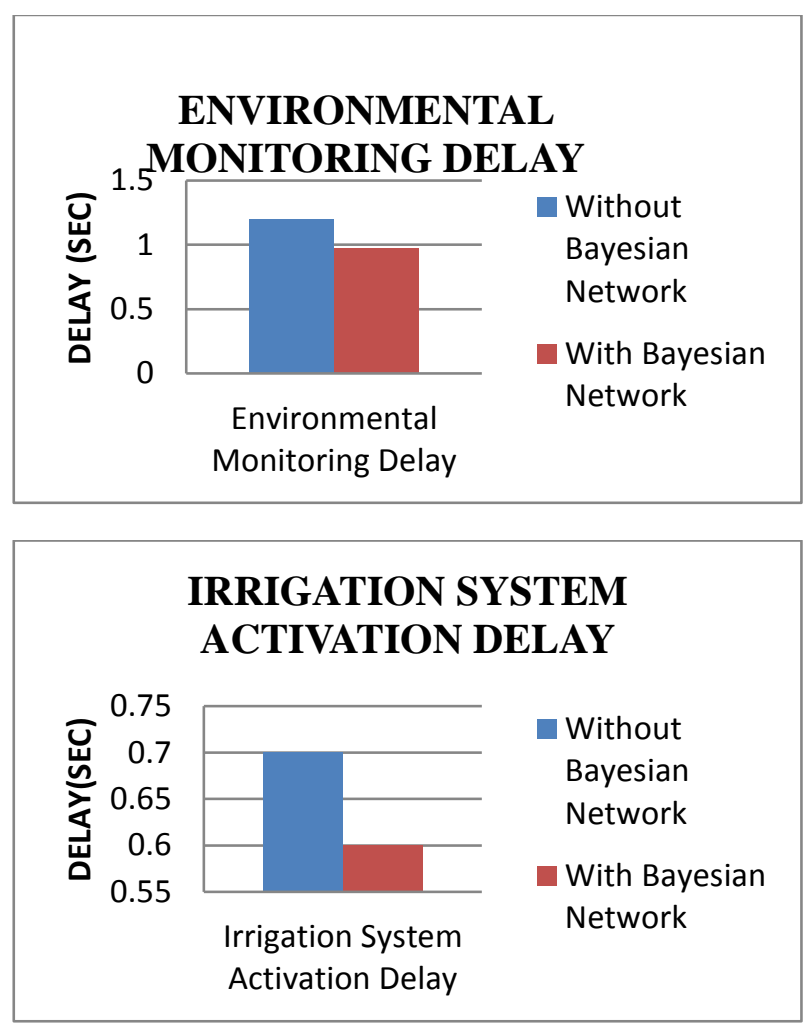

The figure shows performance evaluation of the proposed Bayesian network system compare to the existing monitoring technology in terms of environmental monitoring delay. The metric is calculated by computing the time taken to transfer the command from the device and Response received from the device. The proposed Bayesian network system achieves the better performance with minimized delay in the range of one.

The figure shows performance evaluation of the proposed Bayesian network system compared to the existing monitoring technology in terms of irrigation system activation delay. The metric is calculated by computing the time taken to transfer the command from the device and the response received from the device. The proposed Bayesian network system achieves the better performance with minimized delay in the range of $0.6 \mathrm{~s}$.

\section{Conclusion}

The proposed system in this paper forsupply the optimal amount of water to the crops.Automatic irrigation will help the farmer to cultivate more crops in good quality. The system has a distributed wireless network of soil-moisture and temperature sensors placed in the root zone of the plants. An algorithm was developed with threshold values of temperature and soil moisture that was programmed into a microcontroller-based gateway, to control the water 
quantity. By using the low-cost Wi-Fi radio communication, Communication signals from the sensorand irrigation controller were successfully interfaced.An irrigation machine was converted to be electronically controlled by a programming logic controller, which will update the sensed information. The impact of the IoT on the evolution towards next generation, smart environments will largely depend on the efficient integration of IoT and cloud computing technologies.

\section{References}

[1] A decision support system for managing irrigation in agricultureH.Navarro-Hellian, J.Martinez-del-Rincon, R.Domingo-Miguel,F.Soto-Valles,R.Torres-Sanchez.

[2] Wireless Sensor Network Based Automated Irrigation and Crop Field Monitoring System-G.Nisha, J.Megala-Sixth International Conference on Advanced Computing (ICoAC)-2014.

[3] Agricultural Crop monitoring using IOT- Dr. D.K.Sreekantha, Kavya. A.M- 11th International Conference on Intelligent Systems and Control (ISCO)-2017.

[4] Analysis of Soil Behaviour and Prediction of Crop Yield using Data Mining Approach-Monali Paul, Santosh K.Vishwakarma, Ashok Verma - International Conference on Computational Intelligence and Communication Networks-2015.

[5] Applying Data Mining Techniques to Predict Annual Yield of Major Crops and Recommend Planting Different Crops in Different Districts in Bangladesh-A.T.M Shakil Ahamed, Navid Tanzeem Mahmood, Nazmul Hossain, Mohammad Tanzir Kabir, Kallal Das, Faridur Rahman, Rashedur M Rahman-IEEE-2015.

[6] S.Thilagamani, N. Shanthi," Object Recognition Based on Image Segmentation and Clustering", Journal of Computer Science,Vol. 7,No.11,pp. 1741-1748, 2011.

[7] S.Thilagamani , N. Shanthi," Gaussian and gabor filter approach for object segmentation", Journal of Computing and Information Science in Engineering,Vol.14,Issue 2, pp. 021006,2014.

[8] Dr.P.Santhi, S.Kiruthika," Lung Based Disease prediction Using Lobe Segmentation Based on Neural Networks", International Journal of Pure and Applied Mathematics", Vol.118, No.8, PP. 499-504, 2018

[9] P.Santhi, R.Vikram," Implementation Of Classification System Using Density Clustering Based Gray Level Co Occurrence Matrix (DGLCM) For Green Bio Technology", International Journal of Pure and Applied Mathematics",Vol.118, No.8,PP. 191195,2018

[10] S.Thilagamani, V.Manochitra,"An Intelligent Region-Based Method for Detecting Objects from Natural Images", International Journal of Pure and Applied Mathematics", Vol.118, No.8, PP.473-478, 2018.

[11] S. Thilagamani, N.Shanthi, "A Novel Recursive Clustering Algorithm for Image Oversegmentation", European Journal of Scientific Research, Vol.52, No.3, pp.430-436, 2011.

[12] S.Thilagamani and S. Uma Mageshwari," Risk appraisal for cardiovascular disease among selected young adult women in Coimbatore, India", Indian Journal of Science and Technology, Vol. 3 , No. 6, PP.672-675, 2010.

[13] Hema.C.R, Paulraj.M.P \& Ramkumar.S, "Classification of Eye Movements Using Electrooculography and Neural Networks", International Journal of Human Computer Interaction, Vol.5 (4), pp.51-63, 2014.

[14] Hema, C. R., Ramkumar, S., \& Paulraj, M. P. , "Idendifying Eye Movements using Neural Networks for Human Computer Interaction", International Journal of Computer Applications, 105(8), pp 18-26, 2014.

[15] S.Ramkumar, K.SatheshKumar, G.Emayavaramban, "EOG Signal Classification Using Neural Network for Human Computer Interaction", International Journal of Computer Theory and Applications, Vol.9(24), pp.223-231, 2016

[16] Ramkumar, Dr.K.Satheshkumar and G.Emayavaramban" Nine States HCI using Electrooculogram and Neural Networks", IJET, Vol. 8(6), pp. 3056-3064, Jan 2017.

[17] S.Ramkumar, K.Sathesh Kumar G.Emayavaramban,” A Feasibility Study on Eye Movements Using Electrooculogram Based HCl" IEEE- International Conference on Intelligent Sustainable Systems, pp.384-388, Dec-2017.

[18] G.Emayavaramban, S.Ramkumar, A.Amudha and K.Sathesh Kumar "Classification Of Hand Gestures Using FFNN And TDNN Networks", International Journal of Pure And Applied Mathematics, Vol.118 (8) Pp. 27-32, Jan 2018.
[19] S.Ramkumar, K.Sathesh Kumar, T.Dhiliphan Rajkumar,M.Ilayaraja, K.Shankar, "A review-classification of electrooculogram based human computer interfaces", Biomedical Research, 29(6), Pp. 10781084, April 2018.

[20] T. Mekala, P. Nandhini," Modified Agglomerative Clustering for Web Users Navigation Behavior", International Journal of Advanced Networking and Applications, Vol. 05, Issue: 01, PP.1842-1846, 2013.

[21] S Saravanan, V Venkatachalam,“Advance Map Reduce Task Scheduling algorithm using mobile cloud multimedia services architecture" IEEE Digital Explore,pp.21-25,2014.

[22] K.Deepa, Y.Naveen Raj, "Enhancing the Performance in WSN using Distributed Tracking Algorithm", International Journal of Pure and Applied Mathematics Vol. 118, No. 9 2018, 717-722.

[23] G.Ranjith, M.Vinoth, "A Unified Approach for Effective Use of Cloud Metering Service", International Journal of Pure and Applied Mathematics Vol. 118, No. 9 2018, 801-806.

[24] P. Santhi, S.Thilagamani," A Survey on Audit Free Cloud Storage via Deniable Attribute Based Encryption", IRA-International Journal of Technology \& Engineering, Vol.5, No.1, PP.1-5, 2016.

[25] K.Sindhanaiselvan, T.Mekala," A Survey on Sensor Cloud: Architecture and Applications", International Journal of P2P Network Trends and Technology (IJPTT), Vol.6, PP.1-6, 2014. 